\title{
Editorial zum Themenschwerpunkt Azetabulumfrakturen
}

Frakturen des Azetabulums sind seltene Verletzungen. In spezialisierten Zentren werden pro Jahr in der Regel nicht mehr als 20-25 dieser Frakturen operativ behandelt. Zugleich stellt die chirurgische Versorgungsproblematik sogar an den versierten Operateur hohe Anforderungen. Die dreidimensionale Anatomie ist komplex, und die Klassifikation erfordert ein gutes räumliches Verständnis. Diagnostik und detaillierte Analyse der spezifischen Frakturmerkmale sind aufwendig und erfordern langjährige Erfahrung in der Beckenchirurgie. In Abhängigkeit von der Frakturmorphologie und -lokalisation (vordere und/oder hintere Pfeiler) sind unterschiedliche Zugänge notwendig. Diese sind - bedingt durch die unmittelbare Nachbarschaft vitaler neurovaskulärer Strukturen - risikoreich. Andererseits ist bekannt, dass funktionelles Endergebnis und Langzeitprognose des Hüftgelenks direkt mit der Qualität der Rekonstruktion korrelieren, so dass hohe Ansprüche an die operative Behandlung bestehen.

Die typischen operativen Zugänge zum Azetabulum sind der vordere ilioinguinale Zugang nach Letournel und der dorsale Kocher-Langenbeck-Zugang. Beide Zugänge wurden in dieser Zeitschrift bereits ausführlich beschrieben. In vorliegenden Schwerpunktheft wird nun auf die spezielle Versorgungsproblematik einzelner Frakturformen eingegangen. Dem interessierten Leser wird deutlich, dass nicht alle Azetabulumfrakturen nach dem gleichen Muster behandelt werden können. Vielmehr ist es unabdingbar, auf die spezifischen Merkmale der einzelnen Frakturtypen einzugehen und frakturindividuelle Repositions- und Fixationstechniken anzuwenden.

In diesem Schwerpunktheft werden die operativen Versorgungsstrategien bei vier häufigen Frakturmorphologien detailliert beschrieben. Ziel dieser Publikationen ist es, dem an Becken- und Azetabulumeingriffen interessierten und auf diesem Gebiet bereits versierten Chirurgen neben einer allgemeinen Beschreibung der Operationstechnik praktische Hinweise und Tricks zur spezifischen operativen Versorgungsproblematik zu bieten.

Im ersten Beitrag wird auf die Frakturen der vorderen Wand und des vorderen Pfeilers eingegangen. Diese Frakturen betreffen ausschließlich den ventralen Anteil des Azetabulums, so dass sie typischerweise über einen ilioinguinalen Zugang stabilisiert werden. Sie werden mit zunehmender Häufigkeit auch bei betagten Patienten beobachtet, so dass die stabile Osteosynthese bei osteoporotischen Knochenverhältnissen eine Herausforderung darstellt.

Der zweite Beitrag befasst sich mit der T-Fraktur. Diese Fraktur betrifft definitionsgemäß sowohl den vorderen wie auch den hinteren Pfeiler. Hochtranstektal verlaufende Frakturen, intraartikuläre Fragmente oder Gelenkflächenimpaktionen erschweren die operative Behandlung. In Abhängigkeit von den spezifischen Frakturmerkmalen können sich operativer Zugang und spezifische Versorgungsstrategie unterscheiden. In dieser Arbeit wird die Osteosynthesetechnik über den vorderen Zugang nach Stoppa und den erweiterten hinteren Zugang mit bigastrischer Trochanterosteotomie beschrieben.

Die Zwei-Pfeiler-Fraktur, die Thema des dritten Beitrags ist, beinhaltet immer eine komplette Dissoziation des Azetabulums vom Beckenring. Des Weiteren sind vordere und hintere Pfeiler voneinander separiert. Die Osteosynthesetechnik ist aufwendig und erfordert eine sorgfältige präoperative Planung.

Im vierten Beitrag wird auf die Fraktur der hinteren Wand eingegangen. Diese vermeintlich „einfache“ Fraktur ist mit einer verhältnismäßig hohen Rate an suboptimalen funktionellen Ergebnissen behaftet. Die spezifischen Merkmale der Fraktur werden ausführlich erläutert, und die operative Technik wird detailliert dargestellt.

Wir wünschen dem Leser eine interessante Lektüre und hoffen, dass die einzelnen Beiträge dem erfahrenen Chirurgen eine nützliche Hilfestellung bei der operativen Versorgung dieser anspruchsvollen Frakturen bieten.

Martin H. Hessmann

Oper Orthop Traumatol 2009;21:235

DOI 10.1007/s00064-009-1800-X 